\title{
Mecanismos de coping utilizados por familiares de pacientes em terapia intensiva
}

\author{
Coping mechanisms used by family of patients in intensive care
}

\author{
Franciele de Melo Leal Dreffs ${ }^{1}$, Eniva Miladi Fernandes Stumm², Eliane Roseli \\ Winkelmann ${ }^{3}$, Liamara Denise Ubessi ${ }^{4}$
}

\begin{abstract}
RESUMO
Introdução: Vivenciar o adoecimento de um ente querido gera inúmeros sentimentos, principalmente se associados à internação em unidade de terapia intensiva (UTI).

Objetivo: Analisar os mecanismos de coping utilizados por familiares para enfrentar a internação hospitalar do paciente em UTI adulto e coronariana.

Materiais e Métodos: Estudo quantitativo, descritivo, transversal, realizado na sala de espera das respectivas unidades, em setembro e outubro de 2011, com oitenta familiares. Instrumentos de coleta de dados: sociodemográficos e inventário de Coping-Jalowiec. Foram respeitados todos os aspectos éticos de pesquisas com seres humanos e o projeto foi aprovado pelo Comitê de Ética, sob o parecer $n^{\circ} 220.1 / 2011$.

Resultados: $63,8 \%$ dos familiares pesquisados são mulheres, 41-70 anos incompletos, casadas, com filhos e católicas. $\mathrm{O}$ estilo de coping mais utilizado foi o sustentativo. A avaliação da confiabilidade do inventário de Coping foi determinada pelo Coeficiente Alfa de Cronbach (0,9221).

Conclusão: Os resultados podem servir de subsídios para qualificar a assistência de enfermagem, extensivo aos familiares que, igualmente, necessitam serem cuidados.
\end{abstract}

Palavras-chave: família; assistência ao paciente; unidades de terapia intensiva; enfermagem; adaptação psicológica.

\begin{abstract}
Introduction: Experiencing the illness of a loved one causes immense feelings, especially if associated with Intensive Care Unit (ICU) admission.

Objective: To analyze the coping mechanisms used by families to face hospitalization of the patient in the adult and coronary ICU.

Materials and Methods: Cross-sectional study, conducted in the waiting room of the ICU with eighty families between September and October 2011. Instruments of data collection: sociodemographic and Coping-Jalowiec inventory. The study followed all ethical aspects regarding research with humans and a project was approved by the Ethics Committee under the protocol $n^{\circ}$. 220.1/2011.

Results: $63.8 \%$ of the relatives included are women, 41-70 years of age, married, with children and catholic. The most frequently used coping style was the sustainable. The reliability assessment of the Coping inventory was determined by the Cronbach alpha (0.9221).

Conclusion: The results may provide subsidies to the qualification of nursing care, as well as the family members, which also need to be taken care.
\end{abstract}

Keywords: family; patient care, intensive care units, nursing, psychological adaptation.

\footnotetext{
${ }^{1}$ Enfermeira. Universidade Regional do Noroeste do Estado do Rio Grande do Sul (UNIJUÍ).

${ }^{2}$ Enfermeira. Mestre em Administração pela Universidade Federal do Rio Grande do Sul (UFRGS). Doutoranda em Enfermagem pela Universidade Federal do estado de São Paulo (UNIFESP). Professora no Departamento de Ciências da Vida, UNIJUÍ.

${ }^{3}$ Fisioterapeuta. Doutora em Ciências da Saúde pela UFRGS. Professora no Departamento de Ciências da Vida, UNIJUÍ.

${ }^{4}$ Psicóloga e Enfermeira. Mestre em Educação nas Ciências pela UNIJUÍ. Professora substituta no Centro de Educação Superior Norte do Rio Grande do Sul (CESNORS) da Universidade Federal de Santa Maria (UFSM).
} 


\section{INTRODUÇÃO}

A doença, na maioria das vezes, é concebida como uma ameaça à vida. Vivenciar o adoecimento de um ente querido, normalmente, gera inúmeros sentimentos, principalmente se a este fato estiver associada à necessidade de internação em Unidade de Terapia Intensiva - UTI. A respectiva unidade é um local que presta assistência a pacientes gravemente enfermos e, nessas condições, pode desencadear estresse, tanto para pacientes quanto para seus familiares. Sensações de tensão e de estresse também vêm sendo estudadas e analisadas em indivíduos meses após a experiência de internação de familiar em UTI. Nessa perspectiva, torna-se indispensável avaliar formas preventivas para minimizar prováveis situações de alteração emocional a curto e longo prazo ${ }^{1}$.

A gravidade clínica do paciente, falta de conhecimento, presença de tubos e sondas associados à impossibilidade de comunicação e limitação de movimentos, são descritos como fatores desencadeantes de estresse e de ansiedade nos familiares, já que nesse momento o paciente está impossibilitado de tomar decisões e essas responsabilidades recaem sobre seus familiares. Daí a necessidade de a equipe de enfermagem estar atenta à situação e à saúde mental desses sujeitos ${ }^{1}$. A família é a principal rede social de suporte ao doente nas diferentes fases de seu tratamento e, nessa relação de contribuição ao paciente, espera-se restabelecer a instabilidade do grupo e evitar a geração de uma segunda ordem de doentes, a família ${ }^{2}$.

A experiência de internação em UTI gera nos familiares uma ruptura brusca em seu modo de viver, afeta sua identidade, autonomia e torna o indivíduo incapaz de escolher, de decidir, opinar ou expressar suas escolhas ${ }^{3}$. Diante dessa situação, a família enfrenta uma fase de instabilidade grupal, mas sua participação é fundamental no processo de cuidado ${ }^{2}$, como indispensável para o enfrentamento da doença pelo próprio doente. $\mathrm{O}$ autor descreve o universo familiar e suas necessidades como de responsabilidade de toda a equipe de saúde, por compartilharem medos, dúvidas e incertezas. Nessa mesma linha de pensamento, novas perspectivas referentes à atenção em saúde incluem a família como integrante do processo de cuidado e de reabilitação. Ao envolver a família nesse contexto a carga emocional do familiar e do próprio paciente é amenizada, de maneira a proporcionar melhor interação entre os integrantes do grupo familiar e a própria equipe de saúde.

A permanência de familiares em sala de espera, no aguardo por notícias de seu ente querido internado, aliado a mudanças inesperadas no seu cotidiano, geram, na maioria das vezes, sentimentos que repercutem no enfrentamento da referida situação. $O$ enfrentamento consiste em um processo amplo de várias dimensões com o objetivo de amenizar resultados frente a situações vivenciadas ${ }^{4}$. O autor se reporta ao coping como a utilização de determinados mecanismos comportamentais frente a situações estressantes, sem tornar nocivo ao organismo humano.

O coping é um instrumento cognitivo e comportamental utilizado pelos indivíduos submetidos às situações estressantes ${ }^{5}$. A estratégia de confronto utilizada pelos indivíduos para lidar com o estressor pode ser uma resposta de coping, independentemente do sucesso ou fracasso obtido nesse processo. Existem, segundo os autores, dois tipos principais de coping: centrado no problema e na emoção. O coping centrado na emoção corresponde a estratégias que derivam de processos defensivos, com o objetivo de modificar 0 significado de determinada situação. Esta estratégia faz com que os indivíduos evitem confronto com situações que gerem ameaça e realizem uma série de manobras cognitivas como fuga, distanciamento, aceitação, entre outras, na tentativa de amenizar os estressores. No coping focalizado no problema as estratégias estão voltadas para a realidade, consideradas formas de enfrentamento mais adaptativas, pois possibilitam modificar as pressões ambientais e até eliminar estressores.

A base de apoio para o enfrentamento de situações de terminalidade com utilização de tratamentos complexos e/ou dolorosos está sustentada na fé e na crença em um ser Superior ${ }^{6}$. O cuidado espiritual necessita ser entendido como parte integrante do cuidado de enfermagem, tanto ao paciente quanto aos seus familiares, não como um fragmento isolado, mas 
como resultado de um aspecto individual e que depende da experiência de vida de cada membro familiar. Considera-se que a interação entre profissionais, familiares e pacientes é importante para uma assistência integral e personalizada e, em especial, ao indivíduo que sofre e necessita ser igualmente cuidado, o familiar. Este cuidado é um desafio para o enfermeiro, pois requer estar presente, saber ouvir, respeitar crenças, valores, mas acima de tudo estar disponível à comunicação $^{7}$, ao encontro de um modelo em que a centralidade é a saúde em todos os processos da vida humana ${ }^{8}$.

Com base nessas considerações, busca-se com esta pesquisa responder a questão: Quais são os mecanismos de coping utilizados por familiares para enfrentar a internação hospitalar do paciente em Unidade de Terapia Intensiva Adulto ou Coronariana? Visando respondê-la, elencou-se o seguinte objetivo: Analisar os mecanismos de coping utilizados por familiares para enfrentar a internação hospitalar do paciente em Unidade de Terapia Intensiva Adulto ou Coronariana.

\section{MATERIAIS E MÉTODOS}

A pesquisa é transversal, com abordagem descritiva e quantitativa, realizada em uma instituição de assistência à saúde, mais especificamente, na sala de espera das UTIs. Participaram da pesquisa oitenta familiares de pacientes. As duas unidades disponibilizam 20 leitos de internação, registrados na central de regulamentação de leitos, com uma média de ocupação de $81 \%$. Aos familiares de pacientes internados são disponibilizados horários de visita das 13 horas e 30 minutos às 14 horas e das 19 horas e 30 minutos às 20 horas.

A sala de espera, local da abordagem aos familiares, está localizada próxima à UTI Adulto, UTI Coronariana, Centro Cirúrgico e Unidade de Recuperação Pós Anestésica, de forma a facilitar trocas de informações e orientações aos familiares que aguardam pela visita.

Todos os familiares de pacientes internados em UTI, nos meses de setembro e outubro de 2011, período estabelecido para a coleta de dados, foram convidados a participar da pesquisa, desde que contemplassem os critérios de inclusão elencados: serem familiares ou sujeitos vinculados à família de pacientes internados em Unidade de Terapia Intensiva há no mínimo 24 horas, tempo considerado mínimo para que a pessoa assimile a situação e ter mais de 18 anos de idade. Ressalta-se que a abordagem dos sujeitos foi tranqüila, realizada na sala de espera e sempre que havia mais de um familiar de um paciente, era questionado quem deles iria participar, após explicar os objetivos da pesquisa. Foi entrevistado um familiar de cada paciente e a escolha de qual deles participaria era definido por eles. No respectivo período de coleta de dados, três familiares não puderam participar: dois por apresentar dificuldades de interação e comunicação e um por óbito de seu familiar no momento da entrevista.

Os instrumentos de coleta de dados auto-aplicado utilizados foram: dados sociodemográficos e Inventário de Coping-Jalowiec ${ }^{8}$. Este é um instrumento composto por 60 afirmações que, para análise, foram divididas em oito estilos de coping: confrontivo (confronta o problema diretamente - 10 itens), evasivo (evita o problema - 13 itens), otimista (tem pensamentos positivos - 9 itens), fatalista (desesperança em relação ao problema e pessimista - 4 itens), emotivo (responde emocionalmente - 5 itens), paliativo (passa pelo problema fazendo coisas que se sinta melhor - 7 itens), sustentativo (utiliza suportes para enfrentar problemas 5 itens), e autoconfiante (uso de estratégias que envolvem seus próprios recursos - 7 itens). Os estilos de coping confrontivo e sustentativo são classificados como coping focado no problema, os demais estilos (evasivo, fatalista, otimista, emotivo, paliativo e autoconfiante) focados na emoção.

A coleta de dados foi realizada de forma a respeitar a privacidade de cada familiar, em um ambiente adequado, privativo (uma sala ao lado da sala de espera) e, ao término da entrevista, eles tinham liberdade de realizar alterações nos itens respondidos, se julgassem pertinentes. Após o término da coleta de dados, os mesmos foram submetidos à análise estatística com uso do software estatístico SPSS. Para a obtenção dos resultados e análise dos dados referentes ao uso do Inventário de Coping-Jalowiec se utilizou a pontuação relativa, que resulta da soma dos valores de todos os itens pontuados dentro da 
subescala, dividido pelo número de itens na subescala, denominada de pontuação de meio. Após, se calculou a pontuação relativa dividindo a pontuação de meio para cada subescala, pela soma das pontuações de meio de todas as subescalas (incluindo aquela da subescala). A seguir, foram comparadas as pontuações relativas de cada subescala, a que apresentou maior pontuação foi considerada como estilo de coping mais utilizado no enfrentamento pelos entrevistados ${ }^{9}$.

Foram construídas distribuições conjuntas de frequência e observadas, simultaneamente, duas variáveis do estudo, isto é, representação das variáveis em tabela cruzada, de maneira a favorecer, identificar, com maior clareza, a relação entre elas. O uso de representações tabulares permite melhor compreensão com relação aos resultados obtidos na pesquisa. Esta forma de exposição dos resultados possibilita condições mais objetivas e favorece a compreensão das informações obtidas ${ }^{10}$. Para o estudo da confiabilidade dos indicadores foi realizada análise por meio do Coeficiente Alfa de Cronbach ${ }^{9}$.

Os aspectos éticos que regem uma pesquisa com pessoas foram todos observados e respeitados ${ }^{11}$. O projeto de pesquisa foi aprovado pelo Comitê de Ética em Pesquisa da Unijuí, sob Parecer Consubstanciado no 220.1/2011. Os familiares que participaram da pesquisa assinaram TCLE em duas vias, ficando uma em poder de cada um deles e outra da pesquisadora.

\section{RESULTADOS}

Participaram 80 familiares de pacientes internados em terapia intensiva de um hospital geral do Noroeste do Estado do Rio Grande do Sul, nos meses de setembro e outubro de 2011.

$\mathrm{Na}$ tabela 1 são apresentadas as características sociodemográficas dos familiares participantes da pesquisa. Nesta se constata que mais da metade $(63,8 \%)$ são mulheres, casadas, $42,5 \%$ com 41 a 70 anos de idade, a maioria possui filhos e professam a religião católica.

Conforme explicitado na metodologia, o Inventário de Coping-Jalowiec permite que as estratégias de enfrentamento sejam classificadas em oito estilos diferentes, de acordo com a elaboração cognitiva e
TABELA 1 - Características sociodemográficas dos familiares de pacientes internados em terapia intensiva.

\begin{tabular}{|c|c|c|}
\hline Característica & $\mathbf{N}$ & $\%$ \\
\hline \multicolumn{3}{|l|}{ Sexo } \\
\hline Feminino & 51 & 63,8 \\
\hline \multicolumn{3}{|l|}{ Idade } \\
\hline 18 a 30 anos & 17 & 21,3 \\
\hline 31 a 40 anos & 15 & 18,7 \\
\hline 41 a 50 anos & 14 & 17,5 \\
\hline 51 a 60 anos & 24 & 30,0 \\
\hline 61 a 70 anos & 10 & 12,5 \\
\hline \multicolumn{3}{|l|}{ Estado Civil } \\
\hline Casado (a) & 51 & 63,8 \\
\hline Solteiro (a) & 21 & 26,3 \\
\hline Divorciado (a) & 6 & 7,5 \\
\hline Viúvo (a) & 2 & 2,5 \\
\hline \multicolumn{3}{|l|}{ Escolaridade } \\
\hline Fundamental completo & 15 & 18,7 \\
\hline Fundamental incompleto & 24 & 30,0 \\
\hline Médio completo & 19 & 23,8 \\
\hline Médio incompleto & 6 & 7,5 \\
\hline Superior completo & 8 & 10,0 \\
\hline Superior incompleto & 8 & 10,0 \\
\hline \multicolumn{3}{|l|}{ Religião** } \\
\hline Católica & 56 & 71,8 \\
\hline Evangélica & 22 & 28,2 \\
\hline \multicolumn{3}{|l|}{ Filhos } \\
\hline Sim & 57 & 71,3 \\
\hline \multicolumn{3}{|l|}{ Número de filhos* } \\
\hline Um & 17 & 30,9 \\
\hline Dois & 18 & 32,7 \\
\hline Três & 14 & 25,5 \\
\hline Quatro & 4 & 7,3 \\
\hline Cinco & 2 & 3,6 \\
\hline
\end{tabular}

comportamental do indivíduo. Para avaliar a confiabilidade dos indicadores do Inventário de CopingJalowiec $^{9}$ foi determinado o Coeficiente Alfa de Cronbach. Neste, verificou-se que o índice de consistência interna dos itens da escala do instrumento é de 0,9221, logo, apresenta boa confiabilidade, mostra que o mesmo é preciso e coerente para avaliar os indicadores referidos no questionário.

A avaliação da confiabilidade do instrumento utilizado, Inventário de Coping-Jalowiec, foi feita pelo Coeficiente Alfa de Cronbach. Nesse sentido, a confiabilidade é definida como uma variável ou um conjunto de variáveis capaz de medir o que se propõe, de forma fidedigna e verdadeira $^{12}$. Valores de alfa inferiores a 0,60 podem se 
TABELA 2 - Distribuição da pontuação relativa dos estilos de coping dos familiares de pacientes internados em terapia intensiva.

\begin{tabular}{|c|c|c|c|c|}
\hline \multirow{2}{*}{ Estilos de coping } & \multicolumn{2}{|c|}{ Mais } & \multicolumn{2}{|c|}{ Menos } \\
\hline & $\mathbf{N}$ & $\%$ & $\mathbf{N}$ & $\%$ \\
\hline Confrontivo & 4 & 5,0 & 2 & 2,50 \\
\hline Evasivo & - & - & 6 & 7,50 \\
\hline Otimista & 14 & 17,5 & 1 & 1,25 \\
\hline Fatalista & 1 & 1,25 & 22 & 27,50 \\
\hline Emotivo & - & - & 12 & 15,00 \\
\hline Paliativo & - & - & 5 & 6,25 \\
\hline Sustentativo & 46 & 57,5 & - & - \\
\hline Autoconfiante & 7 & 8,75 & 1 & 1,25 \\
\hline Fatalista e paliativo & - & - & 7 & 8,75 \\
\hline Otimista e fatalista & 1 & 1,25 & - & - \\
\hline Sustentativo e autoconfiante & 1 & 1,25 & - & - \\
\hline Confrontivo e sustentativo & 4 & 5,0 & - & - \\
\hline Evasivo e fatalista & - & - & 1 & 1,25 \\
\hline Fatalista e autoconfiante & - & - & 2 & 2,50 \\
\hline Confrontivo e autoconfiante & - & - & 1 & 1,25 \\
\hline Paliativo e autoconfiante & - & - & 1 & 1,25 \\
\hline Confrontivo e fatalista & - & - & 3 & 3,75 \\
\hline Confrontivo e paliativo & - & - & 4 & 5,00 \\
\hline Confrontivo, otimista e sustentativo & 1 & 1,25 & - & - \\
\hline Fatalista, paliativo e autoconfiante & - & - & 4 & 5,0 \\
\hline Confrontivo, sustentativo e autoconfiante & 1 & 1,25 & - & - \\
\hline Confrontivo, fatalista e autoconfiante & - & - & 1 & 1,25 \\
\hline Confrontivo, paliativo e autoconfiante & - & - & 1 & 1,25 \\
\hline Fatalista, emotivo e paliativo & - & - & 1 & 1,25 \\
\hline Confrontivo, fatalista e paliativo & - & - & 3 & 3,75 \\
\hline Confrontivo, evasivo, fatalista e autoconfiante & - & - & 1 & 1,25 \\
\hline Confrontivo, fatalista, paliativo e autoconfiante & - & - & 1 & 1,25 \\
\hline Total & 80 & 100 & 80 & 100 \\
\hline
\end{tabular}

considerados inadequados; adequados com algumas carências os coeficientes entre 0,60 e 0,70; adequados entre 0,70 e 0,80 ; bons entre 0,80 e 0,85 ; e excelentes os superiores a 0,8513. O índice de consistência interna dos itens da escala do instrumento "Inventário de Coping-Jalowiec"9 é de 0,9221, ou seja, apresenta excelente confiabilidade.

Para a análise dos resultados, utilizou-se a pontuação relativa (PR), a qual demonstra o quanto são considerados os esforços de coping de uma pessoa dentro de um determinado estilo. Com base nessas considerações, verifica-se, na tabela 2 , que o estilo de coping com maior somatório de PR dos familiares pesquisados é o sustentativo, utilizado por 46 deles, seguido do otimista, por 14 e do autoconfiante, 7 indivíduos. Considera-se importante pontuar que os 
estilos que apresentaram menor somatório de PR são, respectivamente, o fatalista, 22 indivíduos e o emotivo por 12 pesquisados.

$\mathrm{Na}$ comparação das pontuações relativas de cada subescala dos familiares participantes da pesquisa, destaca-se que a maior pontuação foi considerada o estilo de coping mais utilizado no enfrentamento aos estressores. Assim, quanto à utilização dos estilos para cada participante do estudo, observou-se o maior percentual para o sustentativo $(57,7 \%)$ seguido do otimista $(17,5 \%)$. Os estilos utilizados pelos familiares dos pacientes, com menores percentuais foram o fatalista $(27,5 \%)$ e o emotivo (15\%).

\section{DISCUSSÁo}

No que tange as características sociodemográficas dos familiares participantes dessa pesquisa, o fato de mais da metade ser mulher, mostra que elas assumem a função de cuidadoras. Historicamente ela é cuidadora, primeiramente junto à família, como mãe, esposa, filha e irmã. A atribuição da maternidade concede a ela a função de cuidadora aos que necessitam de sua atenção, seja no plano físico ou psíquico. Do mesmo modo, é avaliado seu papel, inclusive, em suas escolhas profissionais. Pesquisa que identificou o perfil dos cuidadores de pacientes oncológicos atendidos no período de um ano numa Organização Não Governamental em Brasília. Contou com 53 participantes, 48 do sexo feminino ${ }^{15}$. Um dos resultados do estudo mostra que ocorreu predomínio das mulheres, entre as profissões vinculadas ao cuidado, com ênfase na enfermagem.

No que diz respeito à religião, o fato de a maioria ter uma crença é um fator positivo, no sentido de ajudá-las a lidar melhor com a situação vivenciada. A religião não evita que a pessoa fique menos ansiosa, mas a ajuda a conviver com a ansiedade ${ }^{16}$. Este sentimento está associado ao estresse, tensão ou desconforto psicológico e pode permanecer por instantes ou perdurar por longos períodos de tempo, depende da forma como se lida com eles. Nesse contexto, as manifestações de religiosidade e espiritualidade de famílias de crianças com insuficiência renal crônica, em programa de diálise peritoneal, com a participação de 14 sujeitos, acompanhados durante seis meses, a religião e espiritualidade representam recursos importantes para os familiares no enfrentamento da doença ${ }^{17}$. A religião como um instrumento que promove interação social, pois além de integrar os membros da família, pessoas adeptas a uma religião em razão de suas crenças e princípios apreendidos pela mesma envolvem outras pessoas que necessitam e podem compartilhar de suas necessidades e experiências e reconhecem na ajuda ao próximo uma forma de amenizar seus medos e suas ansiedades $^{17}$.

O uso do Inventário de Coping-Jalowiec mostrou que os familiares dos pacientes internados em terapia intensiva utilizam, predominantemente, o estilo sustentativo, isolado ou associado a outros estilos, seguido do otimista e do autoconfiante. Pesquisas que também utilizaram o respectivo instrumento, identificaram estratégias de enfrentamento que os pacientes utilizavam diante à indicação de cirurgia cardíaca $^{18}$. O acompanhamento de 24 pacientes submetidos à cirurgia, de julho a outubro de 2008 permitiu constatar o predomínio do estilo de coping sustentativo $^{18}$. A utilização do referido estilo ocorreu de forma individualizada e associada a outros. O uso dos mesmos ocorre, segundo os autores, pelo fato de que os pacientes se esforçam para enfrentar a situação de indicação cirúrgica e, com isso, buscam adaptar-se às situações estressantes e utilizam mecanismos e sistemas de suporte social, profissional e espiritual ${ }^{18}$.

Em síntese, não existe um estilo de coping correto ou errado, existe coping efetivo ou não. Estudo realizado em unidades de internação oncológica de cinco hospitais de São Paulo, com 77 enfermeiros assistenciais, há no mínimo um ano na referida unidade, mostrou prevalência do uso de coping focado na emoção $^{20}$. Neste, o indivíduo busca se reestruturar, amenizar sua carga emotiva e alterar a situação. A pesquisa descreveu estratégias adotadas pela equipe de enfermagem a familiares que acompanhavam membros da família em hospitalização, que consistiu na realização de grupos de apoio e oração, relação solidária com o familiar, formação de vínculo de confiança para ajudar na superação de sentimentos, como de impotência, abandono e outros ${ }^{21}$. 
A construção dessa pesquisa mostra que dos familiares de pacientes internados em terapia intensiva adulto e coronariana, $63,8 \%$ são mulheres, com 41-70 anos de idade, ensino fundamental incompleto $e$ casadas. Elas possuem filhos e professam a religião católica. Quanto aos estilos de coping utilizados por eles para lidar com o estresse vivenciado, o sustentativo prevaleceu, seguido do otimista e do autoconfiante. Esses resultados são corroborados pela literatura, bem como pelo resultado obtido com o uso do Coeficiente Alfa de Cronbach $(0,9221)$. Como limitações do estudo destacam-se o número de participantes, o qual está diretamente relacionado ao curto período de coleta de dados. Sugere-se que mais investigações sejam realizadas sobre essa temática, com um maior número de participantes, possibilitando a realização de inferências.

A confiabilidade do instrumento possibilita que o mesmo seja utilizado por pesquisadores, em outras investigações, com extratos populacionais diferentes, de maneira a ampliar conhecimentos referentes ao enfrentamento das pessoas às diferentes situações a que são expostas no seu cotidiano. Em se tratando, especificamente, dos diferentes espaços de atuação do enfermeiro, pesquisas desse tipo podem contribuir no sentido de qualificar a assistência de enfermagem aos indivíduos, extensivo aos seus familiares e subsidiar de maneira importante as práticas dos enfermeiros que atuam em terapia intensiva. No que tange a ações com familiares de pacientes internados em terapia intensiva, o enfermeiro pode planejar e desenvolver ações de escuta terapêutica, com o intuito de ajudá-los a enfrentar a situação aliada a aquisição e ampliação de conhecimentos.

\section{REFERÊNCIAS}

1. Costa JB, Felicetti CR, Costa CRLM, Miglioranza DC, Osaku EF, Versa GLGS, et al. Fatores estressantes para familiares de pacientes criticamente enfermos de uma unidade de terapia intensiva. J Bras Psiquiatr. 2010;59(3):182-9.

2. Tavares JSC, Trad LAB. Estratégias de enfrentamento do câncer de mama: um estudo de caso com famílias de mulheres mastectomizadas. Ciên Saúde Coletiva. 2010;15(suppl.1):1349-58.

3. Baggio MA, Pomatti DM, Bettinelli LA, Erdmann AL. Privacidade em unidades de terapia intensiva: direitos do paciente e implicações para a enfermagem. Rev Bras Enferm. 2011;64(1):25-30.

4. Telles H, Pimenta AMC. Síndrome de Burnout em agentes comunitários de saúde e estratégias de enfrentamento. Saúde Soc. 2009;18(3):467-78.

5. Lazarus RS, Folkman S. Stress, appraisalandcoping. New York: Springer; 1984.

6. Vila VSC, Rossi LA, Costa MCS. Experiência da doença cardíaca entre adultos submetidos à revascularização do miocárdio. Rev Saúde Pública. 2008;42(4):750-6.

7. Borba LO, Schwartz E, Kantorski LP. A sobrecarga da família que convive com a realidade do transtorno mental. Acta Paul Enferm. 2008;21(4):588-94.

8. Ojeda BS, Strey MN. Saberes e poderes em saúde: um olhar sobre as relações interprofissionais. Rev Ciên Saúde. 2008;1(1):2-8.

9. Galdino JMS. Ansiedade, depressão e coping em idosos [dissertação]. Ribeirão Preto: Universidade de São Paulo; 2000.

10. Motta VT. Bioestatística. Caxias do Sul: Educs; 2006.

11. Brasil. Ministério da Saúde. Resolução 196/96. Diário Oficial da União, Brasília, DF, 16 out. 1996. Brasília, DF, 13 nov. 2000. Seção 1, p. 82.

12. Junior JFH. Análise multivariada de dados. Porto Alegre: Bookman; 2005

13. Prieto G, Muñiz J. Un modelo para evaluar la calidad de los tests utilizados em España. Pap Psicol. [periódico online]. 2000 [capturado 18 out 2011]; (77):[9 telas] Disponível em: http://redalyc.uaemex.mx/pdf/ 778/77807709.pdf.

14. Espada MC, Grau C. Estrategias de afrontamiento en padres de niños con cáncer. Psicooncología. 2012 Jun;9(1):25-40.

15. Araújo LZS, Araújo CZS, Souto AKBA, Oliveira MS Cuidador principal de paciente oncológico fora de possibilidade de cura, repercussões deste encargo. Rev Bras Enferm. 2009;62(1):32-7.

16. Mark BW. Como Deus cura a dor. Rio de Janeiro: Sextante; 2008.

17. Paula ES, Nascimento IC, Rocha SMM. Religião e espiritualidade: experiência de famílias de crianças com insuficiência renal crônica. Rev Bras Enferm. 2009;62(1):100-6.

18. Umann J. Guido LA, Linch GFC. Estratégias de enfrentamento à cirurgia cardíaca. Cien Cuid Saude. 2010;9(1):67-73.

19. Rodrigues RP, Labate RC. Luto de profissionais em uma unidade de transplante de células-tronco hematopoéticas. Rev Ciên Saúde. 2012;5(1):26-32.

20. Rodrigues $A B$, Chaves EC. Fatores estressantes $e$ estratégias de coping dos enfermeiros atuantes em oncologia. Rev Lat Am Enfermagem. 2008;16(1):24-8.

21. Beuter M, Brondani CM, Szareski C, Cordeiro FR, Roso CC. Sentimentos de familiares acompanhantes de adultos face ao processo de hospitalização. Rev Enferm. 201;16(1):134-40.

22. Andolhe R. Stress e coping da equipe de enfermagem no cuidado à mulher com câncer de mama [dissertação]. Santa Maria: Universidade Federal de Santa Maria; 2009.

\section{Endereço para correspondência:}

Eniva Miladi Fernandes Stumm

Rua do Comércio 3000

ljuí/RS - CEP 98700-000

Telefone: +555533327722

E-mail: eniva@unijui.edu.br 